4. International Radio Consultative Committee (CCIR): F. G. Smith and H. M. Smith

5. European Physical Society: Edith A. Müller

\title{
IV. SERVICES AND FUNCTIONS OF THE IAU
}

The text under D., pp. 32 through 38 of IAU Trans. XIIC should be replaced by the following text:

D. THE CENTRAL BUREAU FOR ASTRONOMICAL TELEGRAMS

Smithsonian Astrophysical Observatory, Cambridge, Massachusetts 02138, U.S.A.

Director of the Bureau: Dr B. G. Marsden

Associate Directors: Dr O.J. Gingerich, Dr Z. Sekanina, Dr R. B. Southworth

For more than a century, astronomers have maintained a telegraph and cable network for rapid communication of such celestial news as the discovery of a comet or a nova. When the IAU was established, the Central Bureau for Astronomical Telegrams was placed under its jurisdiction (through Commission 6), initially at the Brussels Observatory, and then for many years at the Copenhagen Observatory. Since 1965 the Bureau has been at the Smithsonian Astrophysical Observatory.

Telegrams or cables can be received any time of day or night at the Smithisonian Observatory. The cable address is SATELLITES, NEW YORK (and it is recommended that messages be specified for transmission VIA WUI), the telex number is 92-1428, and the Western Union address for astronomers in North America is RAPID SATELLITE, CAMBMASS. As far as is practicable, messages should be sent according to the code given in the Appendix. Persons telegraphing or cabling information to the Central Bureau are advised also to send the same (and appropriate additional) information by airmail letter at the same time. In many instances, it is quite sufficient to communicate information to the Central Bureau only by airmail letter.

Information is telegraphed or cabled out by the Central Bureau according to the following arrangements. In North America, telegrams are sent collect via Western Union to any astronomers agreeing to pay for the messages. Europe (except for the Netherlands and the U.S.S.R.), North Africa and the Middle East are served via the Meudon Regional Warning Center of the IUWDS; observatories there (except in Czechoslovakia, Sweden, Norway and Finland, whither the 'astrograms' are relayed via additional Regional Warning Centers) are expected to place a deposit with the Central Bureau. The Netherlands, the U.S.S.R., and also Australia and New Zealand receive the astrograms from SOLTERWARN and thence Regional Warning Centers. The rest of the world (Japan, South America, South Africa) is served by certain observatories that relay the astrograms directly from the Central Bureau.

It is not necessary for observatories to receive all the astrograms. Subscribers should specify which of the standard categories they desire:

A. (ALPHA) Comet* discoveries, brighter than magnitude 12

B. (BETA) Comet* discoveries, fainter than magnitude 12

C. (CHARLY) Comet* observations and brief finding ephemerides (primarily for observers)

D. (DELTA) Precise positions, orbits and ephemerides (primarily for computers)

E. (ECHO) Novae and supernovae, brighter than magnitude 12

F. (FRANCE) Novae and supernovae, fainter than magnitude 12

G. (GAMMA) All others, including planetary phenomena

In addition to the astrograms, the Central Bureau also publishes postcard Circulars. Issued at irregular intervals some 70 to 100 times per year, the IAU Circulars contain confirmation of the

\footnotetext{
*and unusual minor planets.
} 
telegrams, as well as information sent to the Central Bureau by mail only: this information includes observed positions and predicted ephemerides for comets and unusual minor planets; further observations of novae, supernovae and unusual variable stars; predictions of occultations by planets; and other astronomical information that requires rather rapid dissemination. Beyond a certain distance from Cambridge the Circulars are sent by airmail. The 700 or so subscribers are billed for the Circulars in advance, and the accounts are diminished by $20 \notin$ for each Cicrular issued (15 $\$$ for those subscribers in North America who do not require invoices and will keep track of their own accounts).

\section{APPENDIX I. ASTRONOMICAL TELEGRAM CODE}

First groups for all telegrams

\section{DISCOVERER OBJECT OBSERVER AAAAB}

1. The name of the discoverer(s) and/or other designation, such as year and letter for a comet, constellation for a nova, galaxy for a supernova $(\mathrm{N}=\mathrm{NGC}, \mathrm{I}=\mathrm{IC}, \mathrm{M}=\mathrm{Messier}$ ), etc.

2. The type of object (COMET, OBJECT, NOVA, SUPERNOVA, variable star = VSTAR, etc.).

3. The name of the observer(s) and/or computer(s).

4. $\mathrm{AAAA}=$ equinox (assumed to be the mean equinox of the beginning of the year specified).

$\mathbf{B}=1$ for an approximate position;

$=2$ for an accurate position;

$=3$ for orbital elements of an object moving around the Sun;

$=4$ for an ephemeris.

Note: when an ephemeris follows the elements, group 4 is replaced by the word EPHEMERIS, and the epoch of the ephemeris is assumed to be the same as that of the elements; see example 3.

Middle groups for a position

Approximate: CDDEE FFFGH IIJJJ LMMNN PQRRS TUUUU VWWXX

Accurate: CDDEE FFFGH IIJJK KKKLM MNNPP PQRRS TUUUU VWWXX

5. Date of observation:

$\mathrm{C}$ = final digit of year;

$\mathrm{DD}=$ month $(01=$ January, $\ldots, 12=$ December $)$;

$\mathrm{EE}=$ day (U.T.).

6. FFFGH = time of observation in decimals of a day (U.T.).

This group may be omitted entirely in the case of observations of stationary objects.

7. Approximate position:

Right ascension II $^{\mathrm{h}} \mathrm{JJ} \mathrm{m} \mathrm{J}$

Declination $\quad \mathrm{LMM}^{\circ} \mathrm{NN}^{\prime}$

$\mathbf{P}=0$;

Accurate position

Right ascension II $^{\mathrm{n}} \mathbf{J J}^{\mathrm{m}} \mathrm{KK}$ s.KK

Declination $\quad \mathrm{LMM}^{\circ} \mathrm{NN}^{\prime} \mathrm{PP}$ "'P

$\mathbf{L}=$ sign: $\mathbf{2}=$ positive, $1=$ negative.

$\mathrm{Q}=1$ for 'total' magnitude $\left(m_{1}\right)$ for a comet;

$=2$ for 'nuclear' magnitude $\left(m_{2}\right)$ for a comet;

$=3$ for visual $\left(m_{v}\right)$ magnitude for objects of stellar appearance

$=4$ for photographic $\left.\left(m_{\rho g}\right)\right\}$ (including minor planets);

$=5$ for photovisual $\left(m_{p v}\right)$ )

$\mathrm{RR}=$ magnitude; if the magnitude is negative, add 100 ;

$\mathrm{S}=$ appearance, according to the table below; or, if the object is not a comet, $\mathrm{S}=$ tenth of a magnitude. 


\section{Nothing reported Tail $<1^{\circ} \quad$ Tail $>1^{\circ}$ about tail}

Stellar appearance

Nothing reported about appearance of object

Object diffuse, without central condensation

Object diffuse, with central condensation o

1

4

7
2

5

8
3

6

9

8. For comets and minor planets, the daily motion:

In right ascension $T$ UUm $U U$

In declination $\mathrm{V} \mathrm{WW}^{\circ} \mathrm{XX}^{\prime}$

For supernovae, the offset from the nucleus of the parent galaxy:

In right ascension T UUUU"

In declination $\mathrm{V}$ WWXX"

$\mathrm{T}, \mathrm{V}=\operatorname{sign}: 2=$ positive (east or north), $1=$ negative (west or south).

Group 8 may be omitted if unknown or irrelevant.

\section{Middle groups for orbital elements}

\section{CDDEE FFFGH IIII JJJJJ KKKKK TTTTT UUUUU}

5. Date of perihelion passage:

$\mathrm{C}$ = final digit of year;

$\mathrm{DD}=$ month $(01=$ January, $\ldots, 12=$ December $)$;

$\mathrm{EE}=$ day.

6. $\mathrm{FFF}=$ time of perihelion pasage in decimals of a day (E.T.).

$\mathrm{G}=$ time interval in days (rounded to the nearest integer) between the first and last positions used for the computation; $0=10$ days or more.

$\mathbf{H}=$ the number and quality of the observations on which the computation is based, or by which the elements have been checked, according to the following table:

$$
\begin{gathered}
\text { Maximum residuals } \\
>5^{\prime \prime} 1^{\prime \prime}-5^{\prime \prime}<1^{\prime \prime}
\end{gathered}
$$

Fewer than three accurate positions* Three accurate positions

$\begin{array}{lll}1 & 2 & 3 \\ 4 & 2 & 3 \\ 7 & 5 & 6\end{array}$

More than three accurate positions

* Or other factors decreasing the reliability of the orbit, such as

7. IIIIII $=\omega$; approximate, doubtful or unsatisfactorily distributed positions

JJJ?JJ $=\Omega$;

$\mathrm{KKK}: \mathrm{KK}=i$.

8. T.TTTT $=q$ (in A.U.);

U.UUUU $=e$.

The group for $e$ may be omitted if the orbit is parabolic $(e=1)$.

Middle groups for ephemerides

CDDEE (IIJJJ LMMNN 9TTTT 8UUUU) cddee

5. Date of the first position in the ephemeris: 


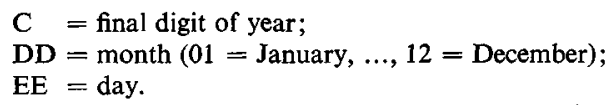

6. It is understood that ephemerides are given for $0^{\text {h }}$ E.T.

7. Right ascension $\mathrm{II}^{\mathrm{h}} \mathbf{J} \mathbf{J} \mathrm{m}$

Declination $\quad \mathrm{L} \mathrm{MM}^{\circ} \mathrm{NN}^{\prime}$

$\mathrm{L}=\operatorname{sign}: 2$ = positive, $1=$ negative.

8. T.TTT = geocentric distance in A.U., preceded by a 9 ;

U.UUU $=$ heliocentric distance in A.U., preceded by an 8.

Groups 7 and 8 are repeated as often as is necessary to complete the ephemeris. Positions should be given at uniform intervals (10,5, 2 or 1 days). Group 8 need not be given for every line in the ephemeris; see examples 3 and 4.

cddee is the date of the last position in the ephemeris, in the same form as before.

\section{Final groups for all telegrams}

\section{YYYYY ZZZZZ REMARKS COMMUNICATOR}

9. YYYYY = the last five figures of the sum of all the groups of digits including and following group 4 (containing the equinox).

$\mathrm{ZZZZZ}=$ the last five figures of the sum of the groups giving the right ascension, declination and magnitude only (or $\omega, \Omega$ and $i$ in the case of orbital elements). In practice, this 'second checksum' includes only the items under group 7 (or involving the letters I through S).

Note: any digit that is unknown, not significant, or otherwise to be withheld should be replaced by a slash $(/)$; this is to count as zero in the summations. A group of five slashes $(/ / / / /)$ should be avoided. If two or more observations are being telegraphed in the same message, checksums should follow each one. A checksum of all numerical information is useful even if the telegram is not sent in code.

10. Any additional remarks in qualification of the observation or the computation. In the case of an accurate position the location of the observing station could be specified.

11. The name of the communicator(s).

\section{Examples}

1. CLARK COMET CLARK $195013061066 / / / 205401313001135$ 2015/ 100028106834805 GILMORE

Gilmore reports that Clark has discovered and observed a comet as follows:

$\begin{array}{cccc}1973 \text { U.T. } & \alpha_{1950} & \delta_{1950} & m_{1} \\ \text { June 10.66 } & 20^{\mathrm{h}} 54 \mathrm{~m} 0 & -31^{\circ} 30^{\prime} & 13\end{array}$

Object diffuse without central condensation, tail $<1^{\circ}$. Daily motion: $\Delta \alpha=+1 \stackrel{\mathrm{m}}{5}, \Delta \delta=-2^{\prime}$.

2. BALLY CLAYTON 1968D COMET ROEMER SCHREUR 19502808272024618513 3362322222821577709056515195028082720872185131682322225 7//// 4876225761 CATALINA LPL

The Lunar and Planetary Laboratory communicates the following observations of comet Bally. Clayton (1968d), obtained by Roemer and Schreur at the Catalina station:

$\begin{array}{cccc}1968 \text { U.T. } & \alpha_{19 s 0} & \delta_{1950} & m_{2} \\ \text { Aug. } 27.20246 & 16^{\mathrm{h}} 51^{\mathrm{m}} 33 \mathrm{~s}: 36 & +32^{\circ} 22^{\prime} 22^{\prime \prime} 8 & 15 \\ 27.20872 & 185131.68 & +322225.7 & \end{array}$

On the first plate the object was diffuse, with central condensation, nothing reported about a tail

3. 1972F COMET CANDY 195032032772656257711595912369092757586054099 EPHEME. RIS 2040300158144339117180934005581474101503150079096180972030001504220418 4930164442 CANDY 
Candy reports that he has calculated parabolic elements and an ephemeris for comet 1972f, as shown below. The elements are based on 3 accurate observations covering an arc of 5 days, and the residuals are all less than $1^{\prime \prime}$.

\begin{tabular}{|c|c|c|c|c|}
\hline \multirow{2}{*}{\multicolumn{5}{|c|}{$\begin{array}{l}T=1972 \text { Mar. } 27.726 \text { E.T. } \\
q=0.9275 \text { A.U. }\end{array}$}} \\
\hline & & & & \\
\hline 1972 E.T. & $\alpha_{1950}$ & $\delta_{1950}$ & $\Delta$ & $r$ \\
\hline Apr. 3.0 & $0^{\mathrm{h}} 15 \mathrm{~m} 8$ & $-44^{\circ} 33^{\prime}$ & 1.171 & 0.934 \\
\hline 8.0 & 0558 & -4741 & & \\
\hline 18.0 & 150.3 & -5007 & 0.961 & 0.972 \\
\hline 18.0 & 300.0 & -5042 & & \\
\hline
\end{tabular}

4. KOHOUTEK OBJECT AKSNES $19504 \quad 11125004121141190325811850036211543$ 003161170900272118320023111950001921210390344811140015712213112076950784703 APOLLO TYPE ASTEROID MAGNITUDE SEVENTEEN SEKANINA

Sekanina communicates the following ephemeris by Aksnes for the object discovered by Kohoutek. The object is an Apollo-type asteroid.

\begin{tabular}{|c|c|c|c|c|c|c|}
\hline 1971 & E.T. & $\alpha_{1950}$ & $\delta_{1950}$ & 4 & $r$ & Mag \\
\hline Nov. & 25.0 & $0^{\mathrm{h}} 41^{\mathrm{m}} \cdot 2$ & $-14^{\circ} 11^{\prime}$ & 0.325 & 1.185 & 17 \\
\hline & 27.0 & 036.2 & -1543 & & & \\
\hline & 19.0 & 0316 & -1709 & & & \\
\hline Dec. & 1.0 & 0272 & -1832 & & & \\
\hline & 3.0 & 023.1 & -1950 & & & \\
\hline & 5.0 & $\begin{array}{lll}0 & 192\end{array}$ & -2103 & 0.344 & 1.114 & \\
\hline & 7.0 & $\begin{array}{lll}0 & 15 & 7\end{array}$ & -2213 & & & \\
\hline
\end{tabular}

5. HONDA SERPENS NOVA HONDA 1900100215 8//// 1825720238030534076441548 BRIGHTNESS INCREASING HIROSE

Hirose repo:ts that Honda has discovered and observed a nova in Serpens, as shown below. The brightness is increasing.

$\begin{array}{rrrr}\text { 1970 U.T. } & \alpha_{1900} & \delta_{1900} & m_{v} \\ \text { Feb. } 15.8 & 18^{\mathrm{h}} 25^{\mathrm{m}} \mathrm{m} & +2^{\circ} 38^{\prime} & 5.3\end{array}$

6. N3811 SUPERNOVA ROSINO $195010920911386247580412 / 20005200038998240264$ ASIAGO

The Asiago Astrophysical Observatory communicates the observation by Rosino of a supernova in NGC $3811\left(\alpha=11^{\mathrm{h}} 38^{\mathrm{m}} \cdot 6, \delta=+47^{\circ} 58^{\prime}\right.$, equinox 1950.0), $5^{\prime \prime}$ east and $3^{\prime \prime}$ north of the nucleus. On 1969 Feb. 9 U.T. the photographic magnitude was 12.

\section{$\checkmark$ PUBLICATIONS OF THE INTERNATIONAL ASTRONOMICAL UNION}

Reference is made to the Report of the Executive Committee, pp. 27-29 and to Information Bulletin Nos. 28, 29 and 30.

\section{SYMPOSIA OF THE INTERNATIONAL ASTRONOMICAL UNION}

Reference is made to the Report of the Executive Committee, pp. 6-7 and to Information Bulletin Nos. 28, 29 and 30. 